\title{
International session 3
}

\section{Sublingual Immunotherapy for Allergic Rhinitis Patients Sensitized to House-Dust Mites}

\author{
Chae-Seo Rhee, MD
}

Department of Otorhinolaryngology, Seoul National University College of Medicine, Seoul, Korea

\begin{abstract}
Allergen-specific immunotherapy (SIT) is a well established treatment option for patients with allergic rhinitis (AR) with or without asthma. Since it was introduced a century ago, SIT has been administered subcutaneously (SCIT), but during 2 last decades, sublingual immunotherapy (SLIT) was gradually introduced in clinical practice, with primary aim of improving safety and convenience. Despite the efficacy of SLIT has been demonstrated in numerous clinical trials and confirmed by several meta-analyses, many countries including the United States does not approve SLIT as a suitable therapeutic option. Some aspects are still needed to being clarification, such as complex mechanism of actions, the optimal duration of SLIT and potential biomarkers for distinguishing responders from non-responders at an early stage of
\end{abstract}

treatment. Also, there have been little data on SLIT in Asian patients with AR sensitized to house dust mites (HDM). I am going to deal with recent studies about impacts of SLIT on HDM sensitized allergic rhinitis patients with the results from our institute; 1) early compliance of conventional escalation SLIT regimen, 2) clinical efficacy of once-daily SLIT without escalation comparing to conventional escalation therapy, 3) clinical efficacy and safety of SLIT in pediatric patients compared to adult patients, 4) the efficacy of SLIT in poly-allergen sensitized patients, 5) the function of Th17 cells in SLIT and IL-17 as a biomarker for response of SLIT. 7) Finally, I would like to provide an evidence of long-term clinical efficacy and safety as well as easily available biomarkers showing surrogacy to clinical response of SLIT. 